\title{
Glycylpeptide N-Tetradecanoyltransferase 1
}

National Cancer Institute

\section{Source}

National Cancer Institute. Glycylpeptide N-Tetradecanoyltransferase 1. NCI Thesaurus.

Code C158397.

Glycylpeptide N-tetradecanoyltransferase 1 (496 aa, $57 \mathrm{kDa}$ ) is encoded by the human NMT 1 gene. This protein is involved in the irreversible transfer of myristoyl groups from tetradecanoyl-CoA to $\mathrm{N}$-terminal glycines of protein substrates. 